\title{
Transcription factor Yin-Yang 2 alters neuronal outgrowth in vitro
}

\author{
Martin Klar $^{1} \cdot$ Pascal Fenske $^{2} \cdot$ Fanny Rezza Vega ${ }^{2} \cdot$ Christof Dame $^{1} \cdot$ Anja U. Bräuer $^{2,3}$
}

Received: 11 March 2015 / Accepted: 6 August 2015 / Published online: 9 September 2015

(C) The Author(s) 2015. This article is published with open access at Springerlink.com

\begin{abstract}
The Yin-Yang 2 (YY2) protein is the most recently described member of the family of YY transcription factors. Despite its high structural and functional homology with the well-characterized YY1, less is known about its role in biological processes. In previous studies, we have found differential yy 2 mRNA expression levels in various cell types of the murine brain. To investigate the functional implication of yy 2 in neurons, we have examined the influence of altered cellular yy 2 concentrations during neuronal differentiation. Our results indicate that both the up- and down-regulation of yy 2 significantly impairs the outgrowth of the major neurite of primary hippocampal neurons and the numbers of neuronal processes in proximate extensions. Moreover, enhanced expression of wild-type yy 2 results in increased cell death,
\end{abstract}

Christof Dame and Anja U. Bräuer contributed equally to this work.

This work was supported by the DFG (BR 2345/1-1 to A.U.B.) and the Sonnenfeld-Stiftung for sponsoring technical equipment (to A.U.B.).

Electronic supplementary material The online version of this article (doi:10.1007/s00441-015-2268-7) contains supplementary material, which is available to authorized users.

Martin Klar

martin.klar@charite.de

Anja U. Bräuer

anja.braeuer@med.uni-rostock.de

1 Department of Neonatology, Charité - Universitätsmedizin Berlin, Augustenburger Platz 1, 13353 Berlin, Germany

2 Institute of Cell Biology and Neurobiology, Center for Anatomy, Charité - Universitätsmedizin Berlin, Charitéplatz 1, 10117 Berlin, Germany

3 Institute of Anatomy, Universitätsmedizin Rostock, Gertrudenstraße 9, 18055 Rostock, Germany whereas elevated expression levels of a yy2 DNA-binding mutant have no effect on cell viability. Therefore, stringent regulation of the cellular yy 2 content might be needed to ensure proper neurite outgrowth and cell vitality.

Keywords Differentiation · Neuron $\cdot$ Cell death $\cdot$ Brain . Morphology $\cdot$ Mouse

\section{Introduction}

During brain development, exact temporal and spatial regulation of cellular processes are essential for the proper functional maturation of neuronal connectivities. Neurite outgrowth, axonal guidance, and dendritic development, all of which occur during neuronal differentiation, are crucial cellular events for the establishment of the neuronal network (da Silva and Dotti 2002). In this context, primary cultured hippocampal neurons are a well-characterized system for studying the molecular mechanisms that regulate changes of neurite morphology (Dent et al. 2004; Schwamborn et al. 2006). In vitro, specific stages of neuronal differentiation are characterized by the following morphological characteristics: after attaching to the substrate neurons form lamellipodia (stage 1), and within a few hours several short undifferentiated processes of similar length, called neurites, are formed (stage 2). After 24-48 h, one of these neurites is selected to become the axon and begins to elongate rapidly, whereas the length of the other neurites is not substantially changed (stage 3 ). Several days later, the remaining neurites begin to grow and differentiate into dendrites (stage 4). The axon and dendrites then reach maturation, and the neurons form synaptic contacts (stage 5; Dotti et al. 1988). The transformation from stage 2 to stage 3 is the crucial step in neuronal differentiation when one neurite becomes the axon, and thereby, the neuron acquires a polar morphology. 
This process involves the coordinated assembly and activation of a number of signaling molecules and their transcriptional regulatory downstream targets to control axon specification.

The biological impact of the transcription factor Yin-Yang 1 (YY1) in neurogenesis has been intensively studied (He and Casaccia-Bonnefil 2008). However, a distinct functional implication of its homolog YY2 in neuronal development has not yet been described. YY2 shares a variety of structural and functional similarities with YY1. Both proteins recognize an identical DNA-binding element with a highly conserved C-terminal C2H2-zinc finger domain (Kim et al. 2007; Luo et al. 2006; Nguyen et al. 2004). Once bound to DNA, these transcription factors can act as activators or repressors (Usheva and Shenk 1996). Depending on the particular target gene, both YY-proteins can mediate either identical or contrary transcriptional activities (Chen et al. 2010; Klar and Bode 2005).

Without specific antibodies against mouse yy2, the cellular content of this protein was initially estimated by analyzing the content of yy2 mRNA. At first, the differential expression pattern of $y y 2$ mRNA in the brain was visualized by in situ hybridization techniques (Luo et al. 2006). For a better understanding of the region- or cell-type-specific $y y 2$ expression, we performed quantitative polymerase chain reaction (qPCR) expression analyses in the developing murine brain and in neurons, astrocytes, and microglia (Drews et al. 2009). Thereby, we identified spatial and temporal differences in $y y 2$ expression levels in the neocortex and cerebellum, supporting the specific regulatory functions of yy 2 within these tissues at particular developmental stages. Moreover, we found lower yy2 levels in neurons, compared with astrocytes or microglia.

In our present study, we show that the length of the major neurite is directly deregulated by knockdown or overexpression of the cellular yy 2 expression. Furthermore, yy 2 overexpression influences the number of neuronal processes in proximate extensions. We have evidence that cofactors are essentially involved in this process. Our experiments imply a functional role of yy 2 for proper neurite development.

\section{Materials and methods}

\section{Animals}

Pregnant, postnatal, and adult C57BL/6 mice, obtained from our central animal facility, were kept under standard laboratory conditions ( $12 \mathrm{~h}$ light/dark cycle; $55 \pm 15 \%$ humidity; $24 \pm$ $2{ }^{\circ} \mathrm{C}$ room temperature [RT] and water ad libidum) in accordance with German and European guidelines (2010/63/EU) for the use of laboratory animals. Approval of experiments was obtained from the local ethical committee of Berlin (LAGeSO: T0108/11).

\section{Plasmid construction}

The mouse yy 2 coding sequence (NCBI accession no. EF688658) was cloned via the $\mathrm{XhoI}$ and BamHI restriction sites into the pCLEG expression vector (Chen et al. 2005). The mouse yy2-FLAG expression plasmid was generated by insertion of a FLAG-linker (5'-cat gga cta caa gga cga cga tga caa gag atc tt- $3^{\prime}$ and $5^{\prime}$-cta gaa gat ctc ttg tca tcg tcg tcc ttg tag tcc atg ggc c-3') into pcDNA3.1(-)muyy2 (Klar and Bode 2005) via ApaI and XbaI restriction sites. The mouse $y y I$ coding sequence (NCBI accession no. NM_009537) was cloned via $B a m H I$ and $E c o R I$ restriction sites into the eukaryotic expression vector pcDNA3.1(-). The resulting plasmids pCLEGmyy 2, pcDNA3.1(-)FLAGmyy 2, and pcDNA3.1(-)myy1 were subsequently verified by sequencing analysis. The yy2 DNA-binding mutant comprising a T312A substitution, derived from human YY1 mutant T348D (Rizkallah and Hurt 2009), was generated by sitedirected mutagenesis with pCLEGmyy2 and the following primer pair: $5^{\prime}$-cag ctt gtt cac get gga gag aaa cc-3' and 5'ggt ttc tct cca gcg tga aca agc tg-3'. Correct amplification was confirmed by Sanger sequencing. For transient knockdown analyses of murine yy2, three different short hairpin RNA (shRNA)-expressing plasmids were constructed. The shRNA sequences were cloned into a BglII/SalI linearized pCGLH vector (Chen et al. 2005): shRNA-yy2-528 (5'-gat ctc gac aac cta cta ttc agt cet gtt caa gag aca gga ctg aat agt agg ttg ttt ttt tgg aac-3' and 5'-tcg agt tcc aaa aaa aca acc tac tat tca gtc ctg tct ctt gaa cag gac tga ata gta ggt tgt cga-3'), shRNA-yy2539 (5'-gat ctc gtt cag tcc tga att tgg aag ctt caa gag agc ttc caa att cag gac tga att ttt tgg aac-3' and $5^{\prime}$-tcg agt tcc aaa aaa ttc agt cct gaa $\mathrm{ttt}$ gga agc tct ctt gaa gct tcc aaa ttc agg act gaa cga-3'), shRNA-yy2-634 (5'-gat ctc gag aga atg gtc aag gtg agc ttt caa gag aag ctc acc ttg acc att ctc ttt ttt tgg aac-3' and 5'-tcg agt tcc aaa aaa aga gaa tgg tca agg tga get tct ctt gaa agc tca cct tga cca ttc tct cga-3'). The numbers in the plasmid nomenclature reflect the corresponding position of the first shRNA base pair within the murine $y y 2$ mRNA sequence (NCBI accession no. NM_001098723). All insertions were verified by sequencing. A pCGLH shRNA expression plasmid against luciferase mRNA (shRNA-luc) was used for control experiments (Franke et al. 2012).

\section{Hippocampal primary neurons: preparation and transfection}

Hippocampal primary neurons were isolated from mouse embryos at day 18 (E18). Hippocampi from several embryos were collected, pooled, and washed twice in ice-cold HBSS (Hank's buffered salt solution, Life Technologies, Darmstadt, Germany). The tissue was incubated in $4 \mathrm{ml} \mathrm{HBSS}$ and $400 \mu \mathrm{l}$ trypsin for $15 \mathrm{~min}$ at $37{ }^{\circ} \mathrm{C}$, resuspended in MEM plating medium supplemented with $10 \%$ horse serum, $0.6 \%$ glucose, 
$100 \mathrm{U} / \mathrm{ml}$ penicillin, and $100 \mu \mathrm{g} / \mathrm{ml}$ streptomycin (all Life Technologies). After dissociation, neurons were plated for immunocytochemical experiments at a density of 100,000 cells/ $\mathrm{cm}^{2}$ onto poly-L-lysine (Sigma-Aldrich, St. Louis, Mo., USA)-coated cover slips in 12-well plates. After 3 h, cells were washed twice with phosphate-buffered saline $(1 \times$ PBS) and incubated in Neurobasal A medium, supplemented with $2 \% \mathrm{~B} 27,0.5 \mathrm{mM}$ glutamine, $100 \mathrm{U} / \mathrm{ml}$ penicillin, $100 \mu \mathrm{g} / \mathrm{ml}$ streptomycin (all Life Technologies) at $37^{\circ} \mathrm{C}$ and $5 \% \mathrm{CO}_{2}$. Primary cells were routinely maintained at $37{ }^{\circ} \mathrm{C}$ with $5 \% \mathrm{CO}_{2}$ in Neurobasal A medium supplemented as described above. Primary neurons were transfected at division 1 (DIV1) by using Effectene (Qiagen, Hilde, Germany) and analyzed $48 \mathrm{~h}$ later (DIV3).

\section{Immunocytochemistry and microscopy}

Primary neurons were fixed $48 \mathrm{~h}$ after transfection in ice-cold $4 \%$ paraformaldehyde with $15 \%$ sucrose in PBS for $10 \mathrm{~min}$ at RT, washed three times with $1 \times$ PBS for $10 \mathrm{~min}$, permeabilized with $0.1 \%$ Triton X-100, $0.1 \%$ sodium citrate in PBS for 3 min at $4{ }^{\circ} \mathrm{C}$, washed again three times with $1 \times$ PBS for $10 \mathrm{~min}$, and incubated for $1 \mathrm{~h}$ with blocking solution, at RT, containing $10 \%$ FCS in $1 \times$ PBS. Neurons were incubated with primary antibodies overnight at $4{ }^{\circ} \mathrm{C}$ and then washed three times with $1 \times$ PBS for $10 \mathrm{~min}$. Antibodies were used at the following dilutions: anti-green fluorescent protein (GFP; AbCam, Cambridge, UK) 1:2500, anti-FLAG (7425; Sigma-Aldrich) 1:1000, and anti-YY2 (A-5; sc-377008; mouse monoclonal antibody; Santa Cruz Biotechnology, Heidelberg, Germany) 1:100 and 1:250, respectively. The secondary antibodies, namely Alexa Fluor 488 and Alexa Fluor 568 at a dilution of 1:1500, were subsequently applied for 90 min at RT. Slides were mounted with Immu-Mount (Thermo Fisher Scientific, Waltham, Mass., USA). Confocal images were acquired with an upright laser microscope (Leica DM 2500) equipped with a $\times 63$ objective (oil immersion 1.4 NA) and a $\times 40$ objective (oil immersion, $1.25 \mathrm{NA}$ ) by using the 488-nm line of an argon-ion laser and the 543-nm line of a helium-neon laser. Background correction and adjustment of brightness and contrast were performed by using Leica confocal software. For co-localization studies, z-stacks of both fluorophores were sequentially acquired and projections averaged.

\section{Morphological analysis}

For morphological analysis, fluorescence images of primary neurons were acquired with an inverted epifluorescence microscope (Olympus IX 81) equipped with a $\times 100$ objective (oil-immersion, 1.3 NA). Images were taken by camera FView II (Olympus) by using cellSens DimensionDesktop Version 1.4.1 (Build 8624) software from Olympus.
Background correction and the adjustment of brightness and contrast were performed by using Adobe ${ }^{\circledR}$ Photoshop CS 3 software. The length of the longest neurite was measured by using Image $1.45 \mathrm{~s}$ software. Data analysis was performed by using GraphPad Prism 5 (GraphPad Software, La Jolla, Calif., USA) on $n=48$ for the control (pCLEG empty vector) and yy 2 wild-type (wt), $n=54$ for yy 2 mut, $n=29$ for shRNA-luc, and $n=33$ for shRNA-yy2-539. Numbers of primary neurites emerging from the cell body were calculated. Data analysis was performed by using GraphPad Prism 5 from $n=46$ for control (pCLEG empty vector) and yy $2 \mathrm{wt}, n=52$ for yy 2 mut, $n=27$ for shRNA-luc, and $n=31$ for shRNA-yy2-539. The number of neurites was determined by means of Sholl analysis, by using $2410-\mu \mathrm{m}$-spaced concentric circles with maximal distances of $250 \mu \mathrm{m}$ distal to the cell body. The innermost circle with a radius of $20 \mu \mathrm{m}$ was placed onto each neuron, with its center roughly coinciding with the center of the soma. Neurite intersections at each circle were manually counted by using ImageJ. Sholl analysis of neurons was performed by using GraphPad Prism 5 on $n=48$ for control (pGLEG empty vector) and yy $2 \mathrm{wt}, n=54$ for yy 2 mut, $n=$ 29 for shRNA-luc, and $n=33$ for shRNA-yy2-539. One investigator, blind to the experimental conditions, performed the measurement. For all measurements, 3-6 different transfections were used. The measurement performed in micrometers or $\mathrm{n}$ was converted to a percentage. Data are reported and presented as means \pm SEM. Statistical significance was determined by using the two-tailed Mann Whitney test for unpaired data at the given significance level $(P: * * * 0.001<P ; * * 0.001$ $<P<0.01 ; * 0.01<P<0.05$ ).

\section{Cell lines and transfection experiments}

N1E-115 mouse neuroblastoma (ATCC CRL-2263), human embryonic kidney HEK293 (DSMZ no.: ACC 305), and human cervix carcinoma HELA (DSMZ no.: ACC 57) cells were grown at $37{ }^{\circ} \mathrm{C}$ in a humidified $5 \% \mathrm{CO}_{2}$-enriched atmosphere in Dulbecco's modified Eagle's medium (DMEM) supplemented with $10 \%$ fetal bovine serum, $50 \mathrm{U} / \mathrm{ml}$ penicillin, and $10 \mathrm{mg} / \mathrm{ml}$ streptomycin (all PAN-Biotech, Aidenbach, Germany). For transfection experiments, cells were plated at a density of 50,000 cells $/ \mathrm{ml}$ per $\mathrm{cm}^{2}$ onto poly-L-lysine (SigmaAldrich)-coated coverslips in 12-well plates. Next day, the medium was renewed, and the cells were transfected with $1 \mu \mathrm{g}$ pCLEG, pCLEG-yy2, or pCLEGmYY2 (T312A) by using lipofection reagent FuGene 6 (Roche, Mannheim, Germany) according to manufacturer's protocol and analyzed $24 \mathrm{~h}$ later. Transfection experiments to validate shRNAexpression constructs were conducted with 200,000 HEK 293 cells per 6-well plate by lipofection with Metafectene (Biontex, München, Germany). One day after plating, cells were transfected with $24 \mu \mathrm{g}$ of the particular pCGLH construct. Next day, cells were transfected with $1 \mu \mathrm{g}$ of the 
corresponding pCGLH and $1 \mu \mathrm{g}$ yy2 expression plasmid pCLEGmyy2, respectively. After $24 \mathrm{~h}$, cells were lyzed in RIPA buffer (0.05 M TRIS, pH 7.4, 0.15 M NaCl, $0.001 \mathrm{M}$ EDTA, 0.001 M EGTA, $1 \%$ Triton-X, $0.25 \%$ [Na]DOC). Ectopic expression of mouse yy 2 wild-type and T312 mutant protein in HELA cells was achieved by using the Metafectene transfection protocol. At 2 days after transfection with $2 \mu \mathrm{g}$ pCLEGmyy2 or pCLEGmyy2 (T312A) plasmid, respectively, cells were lyzed in RIPA buffer.

\section{Western blot analysis}

Whole cell lysates were prepared by lysis in RIPA buffer. Mouse yy2 and yy 1 were in vitro-translated from corresponding pcDNA3.1(-) expression plasmids by using the TNT-Coupled Transcription/Translation system (Promega, Mannheim, Germany) according the manufacturer's protocol. Whole cell lysates or in vitro-translated proteins were separated by SDSpolyacrylamide gel electrophoresis and subsequently transferred by semi-dry blot technique onto nitrocellulose membranes. After the membrane had been blocked overnight in $5 \%$ nonfat dry milk/ $1 \times$ TBS-T (30 mM TRIS-HCl pH 7.6, $150 \mathrm{mM} \mathrm{NaCl}$, $0.1 \%$ Tween 20 ), immobilized proteins were detected by using the following primary and horseradish-peroxidase-conjugated secondary antibodies: anti-FLAG (Sigma-Aldrich; M2; 1:2000), anti- $\beta$-actin (Sigma-Aldrich; 1:5000), anti-GFP (Abcam, 1:5000), anti-YY1 (sc-281; 1:500), anti-YY2 (sc377008; 1:500), anti-mouse (sc-2005; 1:10000), and antirabbit (sc-2004; 1:10,000; all from Santa Cruz Biotechnology). Visualization was conducted with the Western Blotting Luminol Reagent (Santa Cruz Biotechnology).

\section{Propidium iodide staining}

Cell survival was analyzed by using the intercalating agent propidium iodide (PI; Carl Roth, Karlsruhe, Germany). At $24 \mathrm{~h}$ after transfection of N1E-115 cells, the culture medium was replaced with fresh preheated medium containing $5 \mu \mathrm{g} / \mathrm{ml}$ PI and incubated for $1 \mathrm{~h}$ at $37{ }^{\circ} \mathrm{C}$ under a humidified $5 \% \mathrm{CO}_{2}$ enriched atmosphere. Fluorescence images were acquired with the inverted Olympus IX 81, as described above, but equipped with a $\times 20$ objective $(0.45 \mathrm{NA})$. Each experiment was carried out in triplicate and repeated 6-9 times. PI-stained cells were counted by using ImageJ Gel densitometry software. The percentage of dead cells was calculated as a proportion of PIpositive cells to that of total cells. The amount of total cells per well were calculated based on the plated cell number. Data analysis was performed by using GraphPad Prism 5 on $n=27$ for control (pCLEG empty vector), $n=24$ for yy $2 \mathrm{wt}$, and $n=52$ for yy 2 mut. Data are reported and presented as means \pm SEM. Significance was assessed by using the two-tailed Mann Whitney test for unpaired data at the given significance level $(P$ : *** $0.001<P ; * * 0.001<P<0.01 ; * 0.01<P<0.05$.

\section{Quantitative reverse transcription plus PCR}

N1E-115 cells were transiently transfected with empty pCLEG (control), yy $2 \mathrm{wt}$ or yy 2 mut expression plasmids. After $24 \mathrm{~h}$, total RNA was isolated by using the TRIzol protocol (Life Technologies). Subsequently, $2 \mu \mathrm{g}$ RNA were reversely transcribed into cDNA and subjected to qPCR analysis by using the following TaqMan Gene Expression Assays for $\beta$-actin (4352933E), yy2 (Mm03059489_sH), bax (Mm00432051_m1), and bcl2 (Mm00477631_m1; all Life Technologies).

\section{Results and discussion}

\section{YY2 is expressed in immature neurons}

Here, we investigated the functional implication of yy 2 during neuronal differentiation by deregulation of the endogenous yy 2 protein content. To date, the yy 2 transcription factor has not been studied in mice at the protein level at all. Thus, we initially validated the commercially available anti-YY2 mouse monoclonal antibody from Santa Cruz Biotechnology for immunoblots and for immunofluorescence microscopy analysis to visualize altered cellular yy 2 concentrations. Because of the high homology of both yy proteins, especially within the Cterminal DNA-binding zinc finger domain, some purchasable antibodies cross-react between both yy transcription factors (Klar 2010, 2012; Klar and Bode 2005). To establish the specificity of the monoclonal antibody, we performed immunoblots with in vitro-translated yy 2 and yy 1 proteins. As depicted in Fig. 1a, the anti-yy2 antibody exclusively detected yy2, whereas yy1 was only recognized by a specific anti-yy 1 antibody. To investigate further the suitability of this anti-yy2 antibody for immunofluorescence, we transfected hippocampal cultured neurons with expression plasmids encoding for FLAG-tagged yy 2 and performed co-localization studies (Fig. 1b, anti-yy2, b' anti-FLAG, b" merge). This analysis clearly demonstrated that the yy 2 antibody recognized FLAG-yy 2 overexpression in primary neurons.

To examine the effects of an altered cellular content of yy 2 in immature neurons, we generated molecular tools for ectopic expression and knockdown studies of yy 2 optimized for detection by fluorescence microscopy. Next to yy 2 itself or a particular shRNA, all constructs constitutively co-expressed enhanced GFP (eGFP) for the identification of transfected primary neurons. Unfortunately, the reactivity of commercially available antibodies against mouse yy 2 was not sufficient to detect the endogenous level of this transcription factor in whole-cell and nuclear extracts from hippocampal primary neurons by Western blot analysis. To validate the knockdown efficiencies of three different shRNA expression constructs, HEK293 cells were co-transfected with FLAG-tagged mouse 
Fig. 1 Antibody and yy2 knockdown verification. a Immunoblot $(I B)$ analysis for testing reactivity and specificity of the anti-yy2 antibody sc377008 by using in vitrotranslated mouse yy1 and yy2, respectively. Pure reticulocyte lysate served as control. b, b', b" Primary neurons were transfected with FLAG-tagged yy2, and confocal fluorescence microscopy was conducted with indicated antibodies. c

Knockdown efficiency test of three different short hairpin RNA (shRNA) expression plasmids directed against mouse $y y 2$ in HEK293 cells. Co-expression of FLAG-tagged yy2 was used to identify knockdown efficiencies easily. An shRNA expression plasmid against luciferase mRNA (shRNA-luc) and the empty vector construct (control) were used for control experiments. A Western blot against green fluorescent protein $(G F P)$ was performed to compare transfection efficiencies of the shRNA expression plasmids and the immunological detection of $\beta$-actin, which served as a loading control. d, d', d"

Visualization of endogenous yy2 and its efficient knockdown in primary neurons compared with non-transfected and control transfected (e, e', e") cells via confocal fluorescence microscopy. Co-localization analysis of GFP/shRNA expression plasmid and yy 2 was conducted by using the indicated antibodies. The areas in the white boxes are shown at higher magnification below the relevant micrograph (arrow cell of interest). Bars $50 \mu \mathrm{m}$
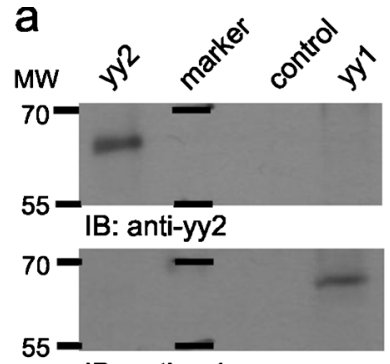

IB: anti-yy1
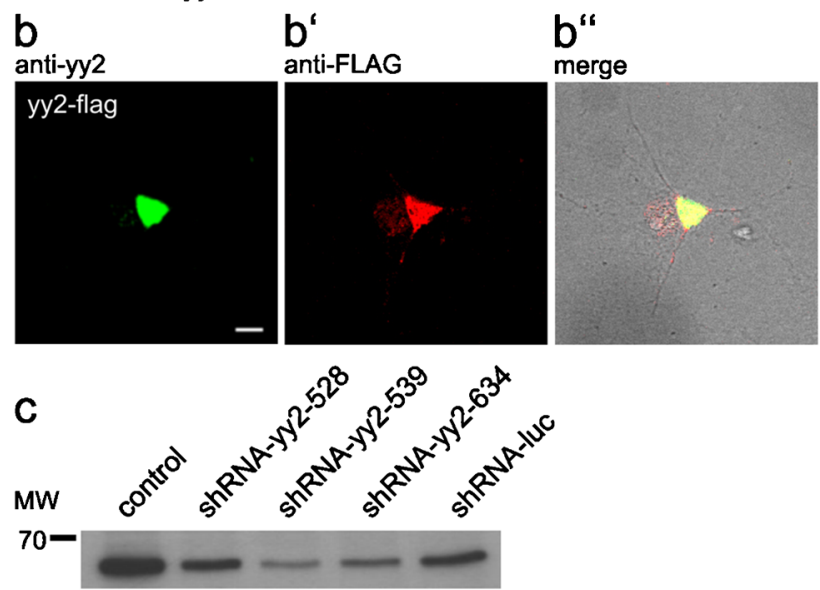

IB: anti-FLAG

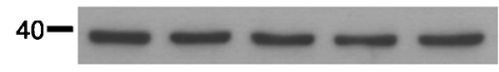

IB: anti-ß-actin

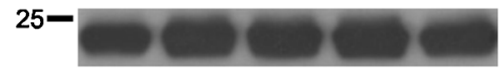

IB: anti-GFP

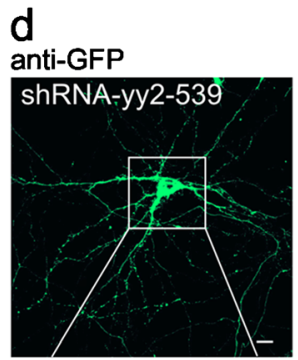

$d^{6}$

anti-yy2

$d^{\prime \prime}$

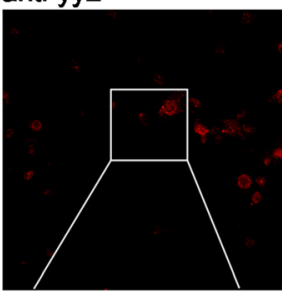

merge

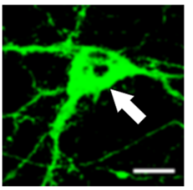

e

anti-GFP

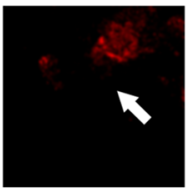

$e^{\prime}$

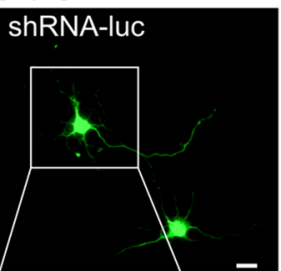

anti-yy2
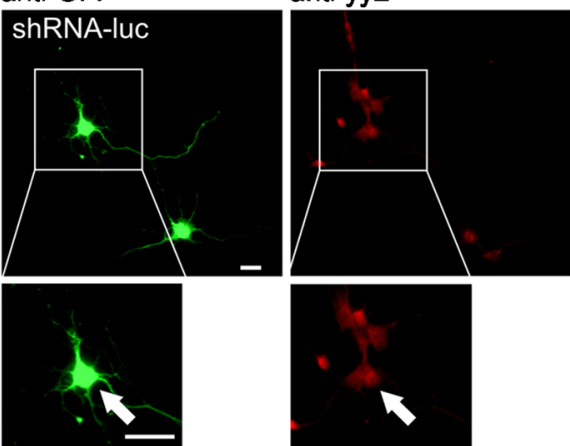

merge
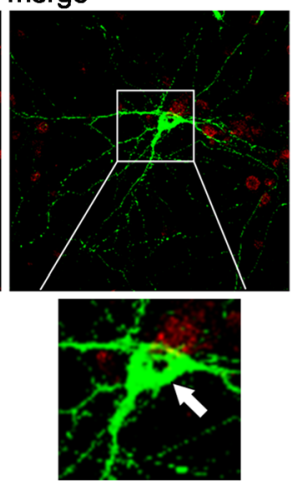

$e^{66}$

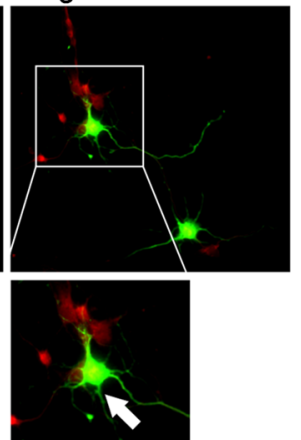


yy2. As shown in Fig. 1c, construct shRNA-539 mediated a significantly reduced expression of FLAG-yy2. Finally, in order to confirm the knockdown capacity of shRNA-539 in primary neurons, we next targeted cellular yy 2 in primary hippocampal neurons. As depicted in Fig. 1d, d', d", we clearly achieved an efficient knockdown of endogenous yy2, whereas all non-transfected neurons or control transfected neurons showed clear nuclear yy2 staining (Fig. 1e, e', e").

Taken together, our data, which were obtained by using a commercially available anti-yy 2 antibody, were able first to demonstrate the specificity of this antibody and second to visualize endogenous yy 2 and its shRNA-driven transient knockdown in primary hippocampal neurons.

\section{Changes of endogenous yy 2 expression influence primary neuron differentiation}

In early, not yet polarized, hippocampal cultured neurons, we investigated whether the expression of yy 2 influenced neurite outgrowth. Initial expression control experiments in HELA cells of both wild-type yy2 (yy2 wt) and a mutant (yy2 mut), which is unable to bind to DNA, revealed proper ectopic protein production (Fig. 2a). In transiently transfected primary hippocampal neurons, yy 2 overexpression resulted in a decrease in the number of neurites projecting to the soma by $20 \%$ (Fig. 2b), whereas overexpression of the mutant yy 2 did not show such changes (Fig. 2b). In addition, the number of neurites projecting to the soma was not significantly altered by the knockdown of yy2 (Fig. 2c). All these findings implied that a normal or reduced endogenous yy 2 concentration did not impact the number of neurites formed by a single neuron, whereas an elevated yy2 level seemed to compete with another transcription factor, possibly YY1, for genomic DNAbinding sites relevant for the regulation of a proper number of neurites. Of note, the latter observation is reminiscent of the effects previously published for yy 2 in reporter gene analyses. In these experiments, some gene promoters were activated in response to lower concentrations of overexpressed yy2, whereas higher yy2 expression level resulted in decreased activities of the same promoters (Nguyen et al. 2004). The transcriptional activities of yy 2 have been proposed to be promoter- and dose-dependent and putatively dependent on other transcription factors such as yy1.

Moreover, a higher expression level of the yy 2 transcription factor leads to a decrease in the length of the longest neurite, which later will become an axon (Dotti et al. 1988). Here, we have demonstrated that the longest neurite reaches only $67 \%$ of the length of the control cells (Fig. 2d, Supplemental Fig. 1a, a'). Interestingly, the overexpression of the yy2 DNA-binding mutant shows an increase of $36 \%$ in the length of the longest neurite compared with the control group (Fig. 2d, Supplemental Fig. 1a"). Moreover, Sholl analyses also revealed substantially reduced numbers of neuronal processes in proximate extensions in yy 2 wt overexpressing primary neurons, whereas the yy2 DNA-binding mutant shows the opposite effect (Fig. 2e, Supplemental Table 1, supplemental Table 3). However, the knockdown of yy 2 also leads to a $37 \%$ increase of the length of the longest neurite compared with that of the control cells (Fig. 2f, supplemental Fig. 1b, b') but does not show significant changes in neurite branching (Fig. 2g, Supplemental Table 2). In contrast to the examinations mentioned above regarding the influence of the altered yy 2 level on the absolute number of neurites, the length of the major neurite is directly deregulated by changes of the cellular yy 2 content. In particular the enhanced length growth of neurites observed in the knockdown experiments underlines the functional role for yy 2 during neuron differentiation, whereas a similar effect achieved by the overexpression of the yy2 DNA-binding mutant suggests that cofactors are essentially involved in the yy2-mediated control of neurite length growth. The excess of mutant yy 2 seems to subdue the interaction between DNA-bound yy2 and its interacting protein partners. So far, only a few yy2-interacting proteins have been described, namely ring finger protein 1 and 2 (RING1, RING2) and embryonic ectoderm development (EED), all of which belong to the family of Polycomb-group (PcG) proteins (Garcia-Tunon et al. 2011). As PcG proteins are known to play a fundamental role mainly in gene silencing, we are tempted to speculate that yy 2 is part of this gene regulatory machinery. Interestingly, knockdown studies of enhancer of zeste 2 (EZH2), which represents next to EED and suppressor of zeste 12 homolog (SUZ12), one of the three core subunits of the polycomb repressor complex 2 (PRC2; Cao et al. 2002) by Wang et al. (2012) have shown that a decreased EZH2 level induces neurite extensions. All these results indicate that changes in the expression level of yy 2 negatively influence neuronal differentiation processes.

\section{YY2 overexpression leads to neuronal cell death}

During our transient yy 2 transfection assays in primary hippocampal neurons, we found many obviously degraded cells when wild-type yy 2 was overexpressed (data not shown). Thus, we asked whether an increase of yy 2 expression specifically forces neuronal cell death. By using the neuroblastoma cell line N1E-115 in order to attain a higher transfection rate compared with primary neurons, we found that yy 2 wt overexpression significantly increased cell death compared with that of controls (control $=14 \%$; yy $2 \mathrm{wt}=34 \%$; Fig. $2 \mathrm{~h}$ ). Interestingly, elevated level of yy 2 mut had no influence on cell death (Fig. 2h). Functionally to associate the increased cell death with apoptosis, we subsequently analyzed, via qPCR, whether the common (anti-) apoptotic genes Bax and $B c l 2$ were deregulated. Although yy 2 wt and yy 2 mut were strongly upregulated, both $B a x$ and $B c l 2$ showed no alterations at all. Thus, the observed wild-type yy2-induced-cell 
Fig. 2 Morphological analyses of primary neurons with altered yy2 expression levels. a Expression control experiments in HELA cells revealed proper ectopic protein expression of wild-type $(y y 2 w t)$ and mutant (yy2 mut) yy2, respectively. Western blot against $\beta$-actin served as loading control. b, $\mathbf{c}$ Comparison of the number of neurites per neuron with respect to yy2 wt or yy 2 mut overexpression and yy 2 knockdown in primary hippocampal neurons. Investigations on the impact of yy2 overexpression levels on the outgrowth of major neurites (d) and the numbers of neuronal processes (e) in primary hippocampal neurons and yy 2 knockdown effects on the outgrowth of the major neurites (f) and the numbers of neuronal processes (g). h PI-staining. Quantification of dead N1E-115 neuroblastoma cells transiently transfected with yy 2 wt and yy 2 mut, respectively a

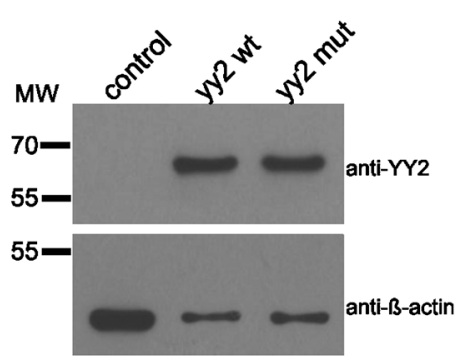

b
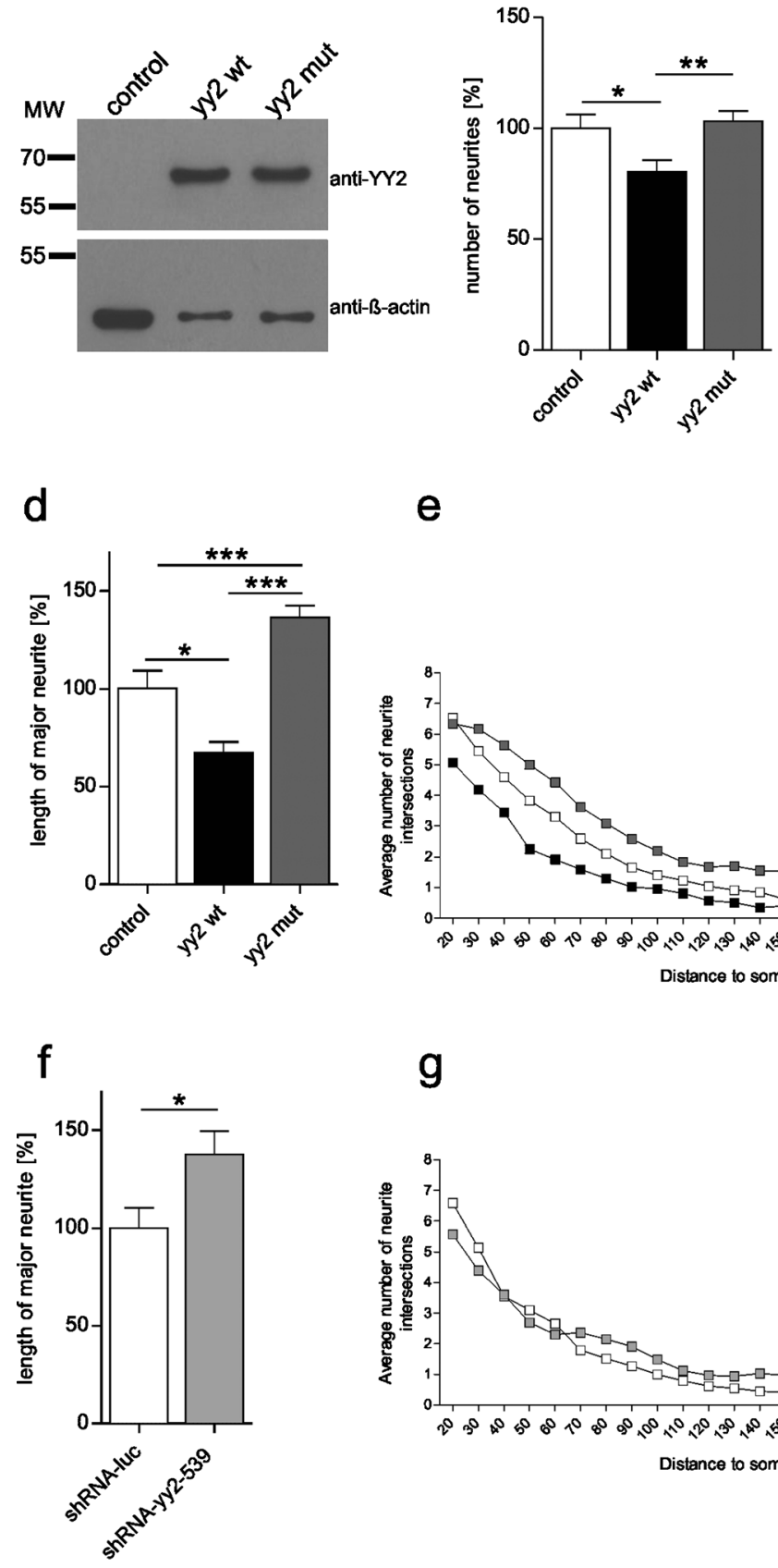

h

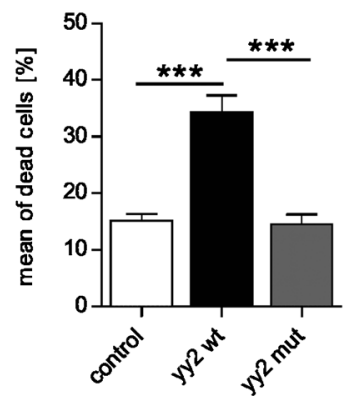

C

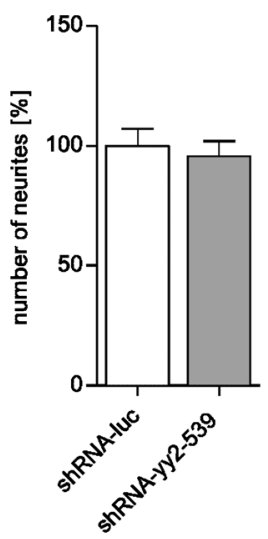

e
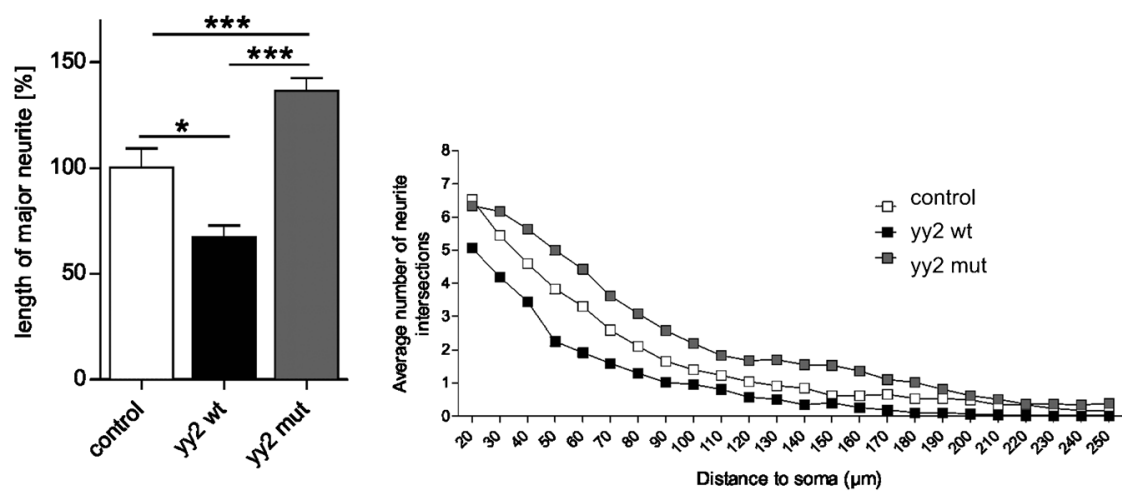

f

d

g

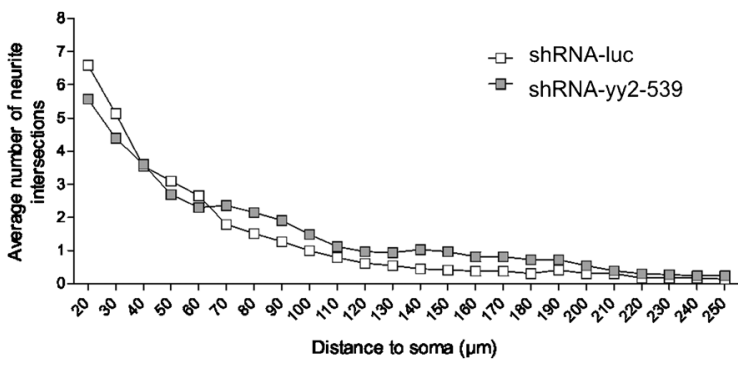


death is not caused by classical apoptosis (Supplemental Fig. 1c). All these findings indicate that an increased yy2 level forces cell death in neuronal cells as a consequence of direct yy2-DNA interaction. We have to assume that an excess of yy2 competes with other transcription factors for functional DNA-binding sites of pivotal genes.

In summary, our experiments support a functional role for yy2 for proper neurite development. A strict regulation of the cellular yy 2 concentrations seems to be needed to ensure normal neurite outgrowth and cell vitality.

Acknowledgments Rike Dannenberg and Jan Csupor are acknowledged for their excellent technical assistance. The authors thank Jutta Schüler for help with the confocal microscopy.

Competing interests The authors declare that they have no competing interest.

Authors' contributions M.K. produced all murine and human YinYang 2 constructs and performed Western blot analysis. P.F. performed immunocytochemistry, co-localization studies, and morphological analyses. F.R.-V. performed the N1-E115 experiments, and A.U.B. and C.D. designed the study. A.U.B., M.K., and C.D. wrote the paper with substantial contributions from all co-authors.

Open Access This article is distributed under the terms of the Creative Commons Attribution 4.0 International License (http:// creativecommons.org/licenses/by/4.0/), which permits unrestricted use, distribution, and reproduction in any medium, provided you give appropriate credit to the original author(s) and the source, provide a link to the Creative Commons license, and indicate if changes were made.

\section{References}

Cao R, Wang L, Wang H, Xia L, Erdjument-Bromage H, Tempst P, Jones RS, Zhang Y (2002) Role of histone H3 lysine 27 methylation in Polycomb-group silencing. Science 298:1039-1043

Chen JG, Rasin MR, Kwan KY, Sestan N (2005) Zfp312 is required for subcortical axonal projections and dendritic morphology of deeplayer pyramidal neurons of the cerebral cortex. Proc Natl Acad Sci U S A 102:17792-17797

Chen L, Shioda T, Coser KR, Lynch MC, Yang C, Schmidt EV (2010) Genome-wide analysis of YY2 versus YY1 target genes. Nucleic Acids Res 38:4011-4026
Dent EW, Barnes AM, Tang F, Kalil K (2004) Netrin-1 and semaphorin 3 A promote or inhibit cortical axon branching, respectively, by reorganization of the cytoskeleton. J Neurosci 24:3002-3012

Dotti CG, Sullivan CA, Banker GA (1988) The establishment of polarity by hippocampal neurons in culture. J Neurosci 8:1454-1468

Drews D, Klar M, Dame C, Brauer AU (2009) Developmental expression profile of the YY2 gene in mice. BMC Dev Biol 9:45

Franke K, Otto W, Johannes S, Baumgart J, Nitsch R, Schumacher S (2012) miR-124-regulated RhoG reduces neuronal process complexity via ELMO/Dock180/Rac1 and Cdc42 signalling. EMBO J 31:2908-2921

Garcia-Tunon I, Guallar D, Alonso-Martin S, Benito AA, Benitez-Lazaro A, Perez-Palacios R, Muniesa P, Climent M, Sanchez M, Vidal M, Schoorlemmer J (2011) Association of Rex-1 to target genes supports its interaction with Polycomb function. Stem Cell Res 7:1-16

He Y, Casaccia-Bonnefil P (2008) The Yin and Yang of YY1 in the nervous system. J Neurochem 106:1493-1502

Kim JD, Faulk C, Kim J (2007) Retroposition and evolution of the DNAbinding motifs of YY1, YY2 and REX1. Nucleic Acids Res 35: $3442-3452$

Klar M (2010) It is not necessarily YY1 - the frequently forgotten YinYang-2 transcription factor. Proc Natl Acad Sci U S A 107:E190 (author reply: E191)

Klar M (2012) Yin Yang 2: the great unknown within the Yin Yang 1 regulatory network. Crit Rev Oncog 16:239-243

Klar M, Bode J (2005) Enhanceosome formation over the beta interferon promoter underlies a remote-control mechanism mediated by YY1 and YY2. Mol Cell Biol 25:10159-10170

Luo C, Lu X, Stubbs L, Kim J (2006) Rapid evolution of a recently retroposed transcription factor YY2 in mammalian genomes. Genomics 87:348-355

Nguyen N, Zhang X, Olashaw N, Seto E (2004) Molecular cloning and functional characterization of the transcription factor YY2. J Biol Chem 279:25927-25934

Rizkallah R, Hurt MM (2009) Regulation of the transcription factor YY1 in mitosis through phosphorylation of its DNA-binding domain. Mol Biol Cell 20:4766-4776

Schwamborn JC, Li Y, Puschel AW (2006) GTPases and the control of neuronal polarity. Methods Enzymol 406:715-727

Silva JS da, Dotti CG (2002) Breaking the neuronal sphere: regulation of the actin cytoskeleton in neuritogenesis. Nat Rev Neurosci 3:694 704

Usheva A, Shenk T (1996) YY1 transcriptional initiator: protein interactions and association with a DNA site containing unpaired strands. Proc Natl Acad Sci U S A 93:13571-13576

Wang C, Liu Z, Woo CW, Li Z, Wang L, Wei JS, Marquez VE, Bates SE, Jin Q, Khan J, Ge K, Thiele CJ (2012) EZH2 mediates epigenetic silencing of neuroblastoma suppressor genes CASZ1, CLU, RUNX3, and NGFR. Cancer Res 72:315-324 